\title{
Persistent Complete Atrioventricular Block after Induction of General Anesthesia in a Healthy Patient
}

\author{
Byeon Gyeong-Jo, Kim Hye-Jin, Ri Hyun-Su, Lee Su Sung, Kim Hee Young
}

Department of Anesthesia and Pain Medicine, Pusan National University Yangsan Hospital, Yangsan, Korea

\begin{abstract}
A 38-year-old female patient had bradycardia in the preoperative electrocardiogram (ECG), and she showed severe bradycardia, with the heart rate (HR) under 40 beats per minute (bpm) even after arrival in the operating room. Immediately after endotracheal intubation, ventricular tachycardia with HR over $200 \mathrm{bpm}$ occurred, but it disappeared voluntarily. The surgery was postponed for additional cardiac evaluation because of the persistent severe bradycardia. On postanesthesia day 2, complete atrioventricular (AV) block appeared. We expected spontaneous recovery over 2 weeks, but the complete AV block persisted. A permanent pacemaker was eventually inserted, and the patient was discharged without other complications on day 4 after insertion of the pacemaker. We report this case because complete AV block has commonly occurred in patients with risk factors such as first AV block, secondary AV block, or bundle branch block, but complete AV block has occurred despite the absence of arrhythmia in this patient.
\end{abstract}

Key Words: Atrioventricular block, Bradycardia, General anesthesia

Complete atrioventricular (AV) block may cause hemodynamic instability, and pacemaker insertion may be necessary because the condition occasionally does not respond to vagolytic drugs. ${ }^{1}$ Sinus bradycardia has been rarely reported to progress to complete AV block, and it is usually temporary, especially when it is caused by anesthetics. We report a case of a permanent complete AV block that occurred after induction of general anesthesia in a patient who had no history of an atrioventricular block because complete AV block has commonly occurred in patients with risk factors such as first AV block, secondary AV block, or bundle branch block, 2,3 but complete AV block has occurred despite the absence of arrhythmia in this patient.

\section{CASE}

A 38-year old female patient with a height of $160 \mathrm{~cm}$ and a weight of $50 \mathrm{~kg}$ who has hyperthyroidism and hearing impairment was admitted to the operating room to undergo endoscopic
Corresponding Author: Kim Hee Young, Department of Anesthesia and Pain Medicine, Pusan National University Yangsan Hospital, 20, Geumo-ro, Beomeo-ri, Mulgeumeup, Yangsan 50612, Korea

Tel: +82-55-360-2129 Fax:+82-55-360-2149 E-mail: yuvi1981@naver.com

\section{(c) (1) (2)}

Articles published in Kosin Medical Journal are open-access, distributed under the terms of the Creative Commons Attribution Non-Commercial License (http://creativecommons.org/licenses/by-nc/4.0/) which permits unrestricted non-commercial use, distribution, and reproduction in any medium, provided the original work is properly cited.
Received: Aug. 29, 2018

Revised: Oct. 25, 2018

Accepted: Nov. 21, 2018 
dacryocystorhinostomy. The patient had undergone preoperative evaluation one month previously, and the results of the thyroid function tests and other were normal. Sinus bradycardia with a heart rate (HR) of 46 beats per minute (bpm) was noted in the preoperative electrocardiogram (ECG) (Fig. 1). She had undergone endoscopic dacryocystorhinostomy under general anesthesia 5 years ago at the same hospital. During that operation, the preoperative ECG showed a normal sinus rhythm (HR, $61 \mathrm{bpm})$, and there were no complications in the perioperative period. Although bradycardia was observed in the current preoperative ECG, the elective surgery was initiated without additional examinations such as a transthoracic echocardiogram because the patient had no concomitant disease and no symptoms related bradycardia, such as dyspnea, dizziness, and syncope.

Before induction, the patient showed severe bradycardia (HR, under $40 \mathrm{bpm}$ ) and ventricular premature contraction (VPC). There was no specific symptom related to the severe bradycardia, and noninvasive blood pressure (NIBP) was 135/59 mmHg. After intravenous administration of $0.5 \mathrm{mg}$ of atropine, HR increased to $64 \mathrm{bpm}$ and endotracheal intubation was subsequently performed under intravenous bolus administration of thiopental $200 \mathrm{mg}$, rocuronium $40 \mathrm{mg}$, and intravenous infusion remifentanil $25 \mathrm{mcg}$ for 5 minutes. HR decreased to 46 bpm after administration of remifentanil. Anesthesia was maintained with 1.5 vol\% of sevoflurane until endotracheal intubation. Immediately after endotracheal intubation, ventricular tachycardia (VT) with HR over 200 bpm occurred, but NIBP was 165/75 mmHg and bispectral index score was 57. VT disappeared voluntarily after 10 seconds without any treatment and NIBP became 130/85 $\mathrm{mmHg}$. Although the patient showed recovery of sinus rhythm, the surgery was postponed for additional cardiac evaluation because the severe bradycardia (HR, 35-40 bpm) and frequent VPC persisted. During emergence, the HR and BP remained steady at 35-40 bpm and 100-110/50-90 mmHg, respectively. On ECG, the HR was 31-36 bpm and a 2:1 second-degree AV block was observed in the postanesthesia care unit (Fig. 2). NIBP was maintained at 95-124/52-63 mm Hg. Although intravenous administration of $0.5 \mathrm{mg}$ of atropine temporarily increased the HR to $61 \mathrm{bpm}$, it subsequently decreased to 31-32 bpm.

On postanesthesia day 2 , the cardiac rhythm changed from a 2:1 second-degree AV block to

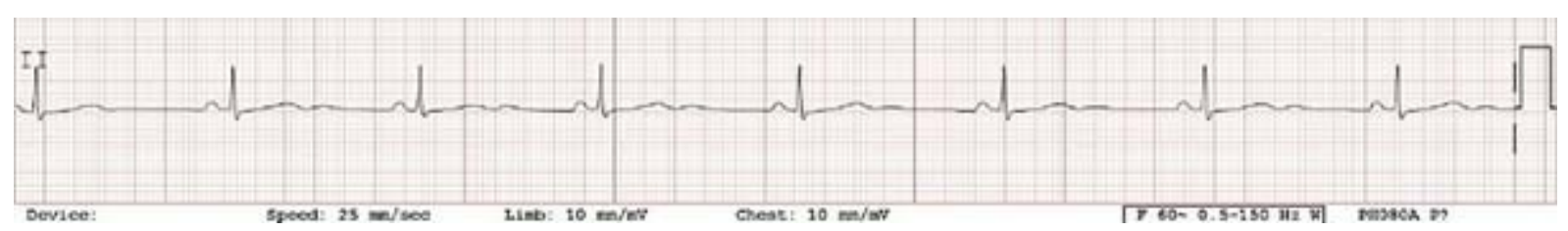

Fig. 1. Preoperative ECG showing sinus bradycardia with heart rate at 46 beats per minute. ECG, electrocardiogram. 


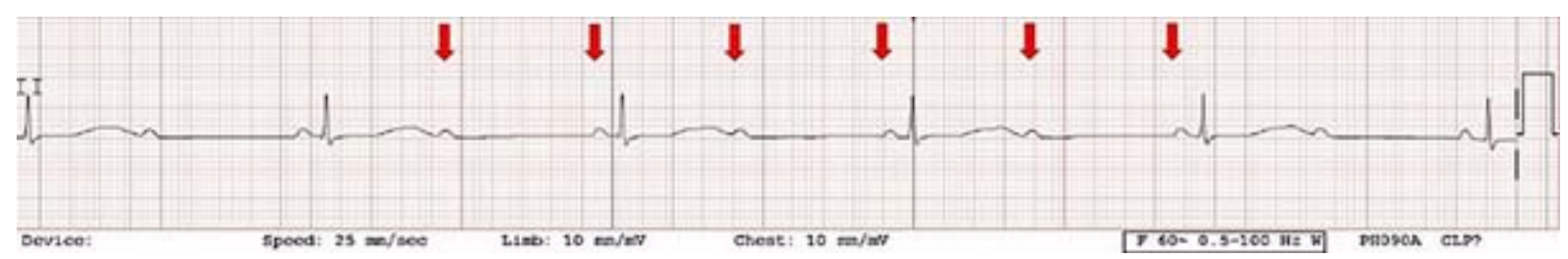

Fig. 2. ECG obtained in the immediate postanesthesia period shows a 2:1 AV block. The red arrows indicate P-waves. ECG, electrocardiogram; AV, atrioventricular.

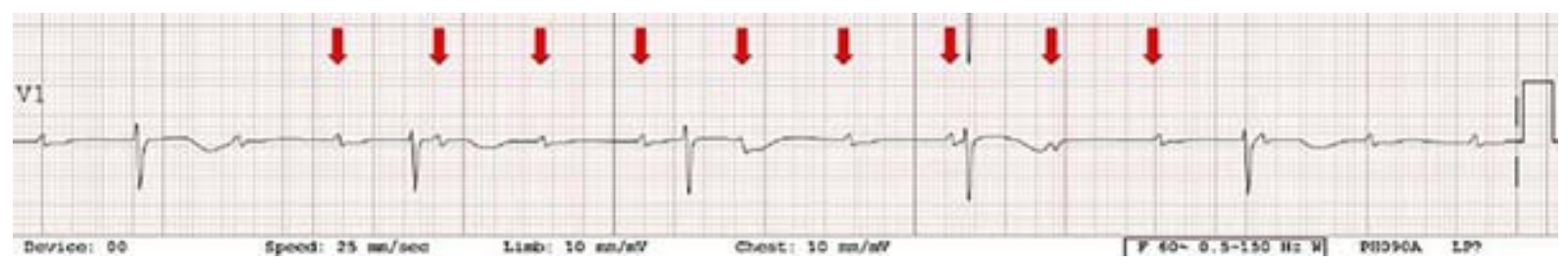

Fig. 3. ECG obtained on postanesthesia day 2 shows a complete AV block. The red arrows indicate P-waves. ECG, electrocardiogram; AV, atrioventricular.

complete AV block with the HR at $30 \mathrm{bpm}$ (Fig 3), but NIBP remained in the normal range and there were no related symptoms. We tried to identify the cause of the AV block by performing laboratory tests of antinuclear antibodies for systemic lupus erythematosus, thyroid hormone, and electrolytes. However, there were no abnormal findings. Transthoracic echocardiographic examination was performed to identify structural abnormalities of the heart, but it showed normal left ventricular size and contractility. The patient also showed no abnormalities in the 24-hour Holter monitoring.

The complete AV block persisted for about 2 weeks; during this period, we continued to monitor the AV block in the hope that it would spontaneously recover to sinus rhythm because we could not exclude sevoflurane and remifentanil as the cause of the complete AV block and the patient was hemodynamically stable. However, a perma- nent pacemaker was eventually inserted, and the patient was discharged without other complications on day 4 after pacemaker insertion.

\section{DISCUSSION}

In a complete AV block, electrical activity in the atrium cannot travel to the Purkinje fibers, and the atrium and ventricle contract individually without any relationship. There are several causes of complete AV block (Table 1), ${ }^{4}$ and complete AV blocks can be congenital or acquired. Congenital complete AV blocks are primarily associated with the maternal antibodies to Ro (SS-A) and La (SS-B) and maternal lupus. ${ }^{5}$ In contrast, acquired complete AV blocks result from various pathologic states causing infiltration, fibrosis, or loss of connection in portions of the healthy conduction system. However, they may also occur af- 
Table 1. Causes of complete AV block

\begin{tabular}{ll}
\hline Most common causes & Very rare causes \\
\hline - Coronary artery disease & - Neuromuscular or neurologic disorders \\
- Degenerative diseases & - Metabolic causes \\
- Nonischemic cardiomyopathies & - Phase IV idiopathic block \\
- Infectious causes & - Radiation-induced \\
- Rheumatic and autoimmune diseases & - Psychiatric conditions \\
- Infiltrative processes & - Unexplained apoptosis of the cardiac -conduction system \\
- Vagally induced & - Acute rheumatic fever \\
- latrogenic causes (including drugs) & - Left ventricular noncompaction \\
& - Thyroid disorders \\
\hline
\end{tabular}

ter administration of AV nodal blocking agents, aortic valve surgery, septal alcohol ablation, percutaneous coronary interventions, or electrophysiological ablation of the slow or fast pathways of the AV node. They can also be caused by neurologic, metabolic, and endocrine diseases in rare cases.

In this case, tests for SS-A/Ro or SS-B/La autoantibodies were performed to assess the possibility of systemic inflammatory and connective tissue diseases, which can cause congenital complete AV blocks. However, the results were negative. Transthoracic echocardiography was also performed to identify structural problems of the heart, and it showed a normal structure.

Although the causes of acquired complete AV blocks are extremely diverse, this patient showed no suspicious symptoms or signs of heart disease, neuromuscular system disease, or infection, and the results of postoperative transthoracic echocardiography and blood tests were normal. On the basis of these findings, neuromuscular diseases, coronary artery disease, cardiomyopathy, and in- fectious diseases were excluded. In rare cases, thyroid disease may be associated with a complete AV block, and this patient had a history of hyperthyroidism, but her thyroid hormone levels were normal. Therefore, we suspected that the newly developed complete AV block was caused by the drugs administered during induction of anesthesia and vagal stimulation caused by procedures such as endotracheal intubation.

Several anesthetics have been reported to cause conduction disturbances, and inhaled anesthetics show dose-dependent effects on myocardial suppression $^{6,7}$ and cardiac conduction systems. ${ }^{8,9}$ Inhaled anesthetics can delay cardiac conduction and inhibit voltage-gated sodium and L-type calcium channels, thereby prolonging the QT interval. ${ }^{10}$ Sevoflurane has depressive effects on the cardiac conduction. ${ }^{11}$ However, sevoflurane is also known to have little or no inhibitory effect on the AV node, and life-threatening arrhythmia associated with its administration has not been reported to date. Sevoflurane shows weaker AV conduction inhibition than halothane or en- 
flurane and similar to that of isoflurane. ${ }^{12}$ In addition, most anesthetics show temporary or reversible courses after their action is finished. Although propofol is reported to be capable of reversing the conduction disturbances caused by sevoflurane, Lee et al. reported that a large bolus of propofol induces a complete AV block. ${ }^{2}$ Opioids such as remifentanil can also cause bradycardia, junctional rhythms, and even temporary sinusoidal arrest. Zaballos et al. suggested that remifentanil depresses sinus node function and most parameters of AV nodal function, which could explain remifentanil-related severe bradyarrhythmias. ${ }^{13}$

In this case, the complete AV block persisted for a considerable amount of time even after stopping general anesthesia using sevoflurane. In other case reports, complete AV block has occurred in patients with risk factors such as first AV block, secondary AV block, or bundle branch block, ${ }^{2,3}$ but complete AV block has occurred despite the absence of arrhythmia in this patient. Therefore, we suggested that persistent complete AV block in this patient was triggered by sevoflurane and remifentanil administration during anesthesia induction in the presence of AV block risk factors such as sinus bradycardia and endotracheal intubation, which induced vagal stimulation. Drugs such as atropine and isoproterenol could be used temporarily to increase heart rate in the patient with complete AV block, but insertion of pacemaker should be considered if complete AV block is persistent. Permanent pacemaker was inserted in this patient under the judgment of the cardiologist because there was no recovery to sinus rhythm although we expected spontaneous recovery for 2 weeks.

Complete AV block may cause serious hemodynamic instability. To avoid such unexpected AV blocks, it is important to preoperatively identify risk factors such as bradycardia that may result in AV blocks even in healthy patients without a notable medical history. Although there are a few rare reports in which sinus bradycardia was converted to a complete AV block after general anesthesia, anesthesiologists should be cautious when administering inhaled and intravenous anesthetics such as propofol and remifentanil because sinus bradycardia may be a part of the sick sinus syndrome and may exacerbate to a heart block, tachyarrhythmia, or alternating bradyarrhythmia. ${ }^{14}$ In addition, careful monitoring and immediate treatment are required when vagal stimulation caused by procedures such as endotracheal intubation can result in bradycardia and AV block. When severe bradycardia with ventricular rate occurs, heart rate should be maintained within appropriate range. If severe bradycardia does not respond to vagolytics such as atropine, pacemaker insertion should be considered according to the guidelines and recommendations for placement of permanent pacemakers in patients with complete AV block which is offered by 2008 American College of Cardiology/American Heart Association/Heart Rhythm Society (ACC/AHA/HRS) (Table 2). ${ }^{15}$ 
Table 2. Indications of permanent pacemaker

\begin{tabular}{|c|c|c|}
\hline $\begin{array}{l}\text { Class I - Indicated } \\
\text { - } 3^{\circ} \text { AVHB: } \\
\text { Symptomatic bradycardia or need for } \\
\text { drugs causing same After catheter } \\
\text { ablation of the arterioventricular } \\
\text { junction } \\
\text { Postoperative and not expected to } \\
\text { resolve } \\
\text { Neuromuscular diseases } \\
\text { Escape rhythm, } 40 \text { beats/min or } \\
\text { asystole }>3.0 \mathrm{~s} \text { in an asymptomatic patient } \\
-2^{\circ} \text { AVHB that is permanent or intermittent, } \\
\text { with symptomatic bradycardia }\end{array}$ & $\begin{array}{l}\text { Class II - May Be Indicated } \\
\text { - Asymptomatic } 3^{\circ} \text { AVHB with average } \\
\text { rate }>40 \text { beats/min } \\
\text { - Type II, } 2^{\circ} \text { AVHB without symptoms } \\
\text { (permanent or intermittent) } \\
\text { - Type I, } 2^{\circ} \text { AVHB at or below His bundle } \\
\text { without symptoms } \\
-1^{\circ} \text { AVHB with symptoms of low cardiac } \\
\text { output that are relieved by temporary } \\
\text { Pacing } \\
\text { - Marked } 1^{\circ} \text { AVHB in a patient with CHF }\end{array}$ & $\begin{array}{l}\text { Class III - Not Indicated } \\
\text { - Asymptomatic } 1^{\circ} \text { AVHB } \\
\text { - Type I, } 2^{\circ} \text { AVHB above His bundle } \\
\text { without symptoms } \\
\text { - AVHB that is expected to resolve }\end{array}$ \\
\hline
\end{tabular}

AVHB, arterioventricular heart block; CHF, Congestive heart failure.

\section{ACKNOWLEDGMENT}

This work was supported by clinical research grant in 2018 from Pusan National University Hospital.

\section{REFERENCES}

1. Epstein AE, DiMarco JP, Ellenbogen KA, Estes NA 3rd, Freedman RA, Gettes LS, et al. ACC/AHA/HRS 2008 Guidelines for Device-Based Therapy of Cardiac Rhythm Abnormalities: a report of the American College of Cardiology/American Heart Association Task Force on Practice Guidelines (Writing Committee to Revise the ACC/AHA/NASPE 2002 Guideline Update for Implantation of Cardiac Pacemakers and Antiarrhythmia Devices): developed in collaboration with the American Association for Thoracic Surgery and Society of Thoracic Surgeons. Circulation 2008;117:e350-408.

2. Noh JI, Lee JH, Woo SY, Kim YK, Cho SH, Kim $\mathrm{SH}$, et al. Complete atrioventricular nodal block after propofol administration in an elderly patient undergoing total knee replacement arthroplasty-A case report-. Korean J Anesthesiol 2013;64:363-6.

3. Shigematsu-Locatelli M, Kawano T, Nishigaki A, Yamanaka D, Aoyama B, Tateiwa H, et al. General anesthesia in a patient with asymptomatic second-degree two-to-one atrioventricular block. JA Clin Rep 2017;3:27.

4. Barra SN, Providência R, Paiva L, Nascimento J, Marques AL. A review on advanced atrioventricular block in young or middle-aged adults. Pacing Clin Electrophysiol 2012;35:1395-405.

5. Kertesz NJ, Fenrich AL, Friedman RA. Congenital complete atrioventricular block. Tex Heart Inst J 1997;24:301-7.

6. Atlee JL 3rd, Rusy BF. Halothane depression of 
A-V conduction studies by electrograms of the bundle of his in dogs. Anesthesiology 1972;36:112-8.

7. Atlee JL 3rd, Rusy BF. Atrioventricular conduction times and atrioventricular nodal conductivity during enflurane anesthesia in dogs. Anesthesiology 1977;47:498-503.

8. Kazama T, Ikeda K. The comparative cardiovascular effects of sevoflurane with halothane and isoflurane. J Anesth 1988;2:63-8.

9. Conzen PF, Vollmar B, Habazettl H, Frink EJ, Peter K, Messmer K. Systemic and regional hemodynamics of isoflurane and sevoflurane in rats. Anesth Analg 1992;74:79-88.

10. Zhou W, Fontenot HJ, Liu S, Kennedy RH. Modulation of cardiac calcium channels by propofol. Anesthesiology 1997;86:670-5.

11. Maruyama K, Agata H, Ono K, Hiroki K, Fujihara T. Slow induction with sevoflurane was associated with complete atrioventricular block in a child with hypertension, renal dysfunction, and impaired cardiac conduction. Paediatr Anaesth 1998;8:73-8.

12. Yokota S. Comparative effects of inhalation anesthetics on atrioventricular conduction with and without calcium entry blockers. Hokkaido Igaku Zasshi 1989;64:43-54.

13. Zaballos M, Jimeno C, Almendral J, Atienza F, Patiño D, Valdes E, et al. Cardiac electrophysiological effects of remifentanil: study in a closed-chest porcine model. Br J Anaesth 2009;103:191-8.

14. Miller RD. Miller's Anesthesia. 8th ed. Philadelphia, USA: Elsevier; 2014. p.1439.

15. Atlee JL, Bernstein AD. Cardiac rhythm management devices (Part I): indications, device selection, and function. Anesthesiology 2001;95:1265-80. 